\title{
The Solution of the Elrod Algorithm for a Dynamically Loaded Journal Bearing Using Multigrid Techniques
}

Claudia M. Woods

Lewis Research Center

Cleveland, Ohio

and

David E. Brewe

Propulsion Directorate

U.S. Army Aviation Research and Technology Activity-AVSCOM

Lewis Research Center

Cleveland, Ohio

(DASA-TH-100941) TEE SCLUTICA OF THE ELBOD

ALGCBITHE FOB A DYNAMICALIY ICADED JODRNAL

EEABING ESING VELTIGRID TECENIGOES (NASA)

$31 \mathrm{E}$

CSCL $13 I$

\section{G3/34 0150179}

Prepared for the

1988 Tribology Conference

cosponsored by the ASME and STLE

Baltimore, Maryland, October 16-19, 1988

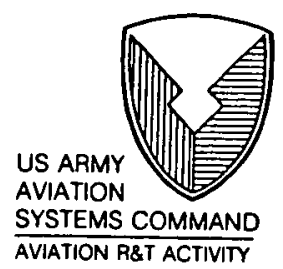


THE SOLUTION OF THE ELROD ALGORITHM FOR A DYNAMICALLY LOADED

JOURNAL BEARING USING MULTIGRID TECHNIQUES

\author{
Claudia M. Woods \\ National Aeronautics and Space Administration \\ Lewis Research Center \\ Cleveland, Ohio 44135 \\ and \\ David E. Brewe \\ Propulsion Directorate \\ U.S. Army Aviation Research and Technology Activity - AVSCOM \\ Lewis Research Center \\ Cleveland, Ohio 44135
}

ABSTRACT

Numerical solution to a theoretical model of vapor cavitation in a dynamically loaded journal bearing is developed, utilizing a multigrid iterative technique. The method is compared with a noniterative approach in terms of computational time and accuracy. The computational model is based on the Elrod algorithm, a control volume approach to the Reynolds equation which mimics the Jakobsson-Floberg and 01 sson cavitation theory. Besides accounting for a moving cavitation boundary and conservation of mass at the boundary, it also conserves mass within the cavitated region via a smeared mass or striated flow extending to both surfaces in the film gap. The mixed nature of the equations (parabolic in the full film zone and hyperbolic in the cavitated zone) coupled with the dynamic aspects of the problem create interesting difficulties for the present solution approach. Emphas is is placed on the methods found to eliminate solution instabilities. Excellent results are obtained for both accuracy and reduction of computational time.

NOMENCLATURE

$A R$ aspect ratio of grid size, $\Delta x / \Delta z$

ed dynamic eccentricity, m 


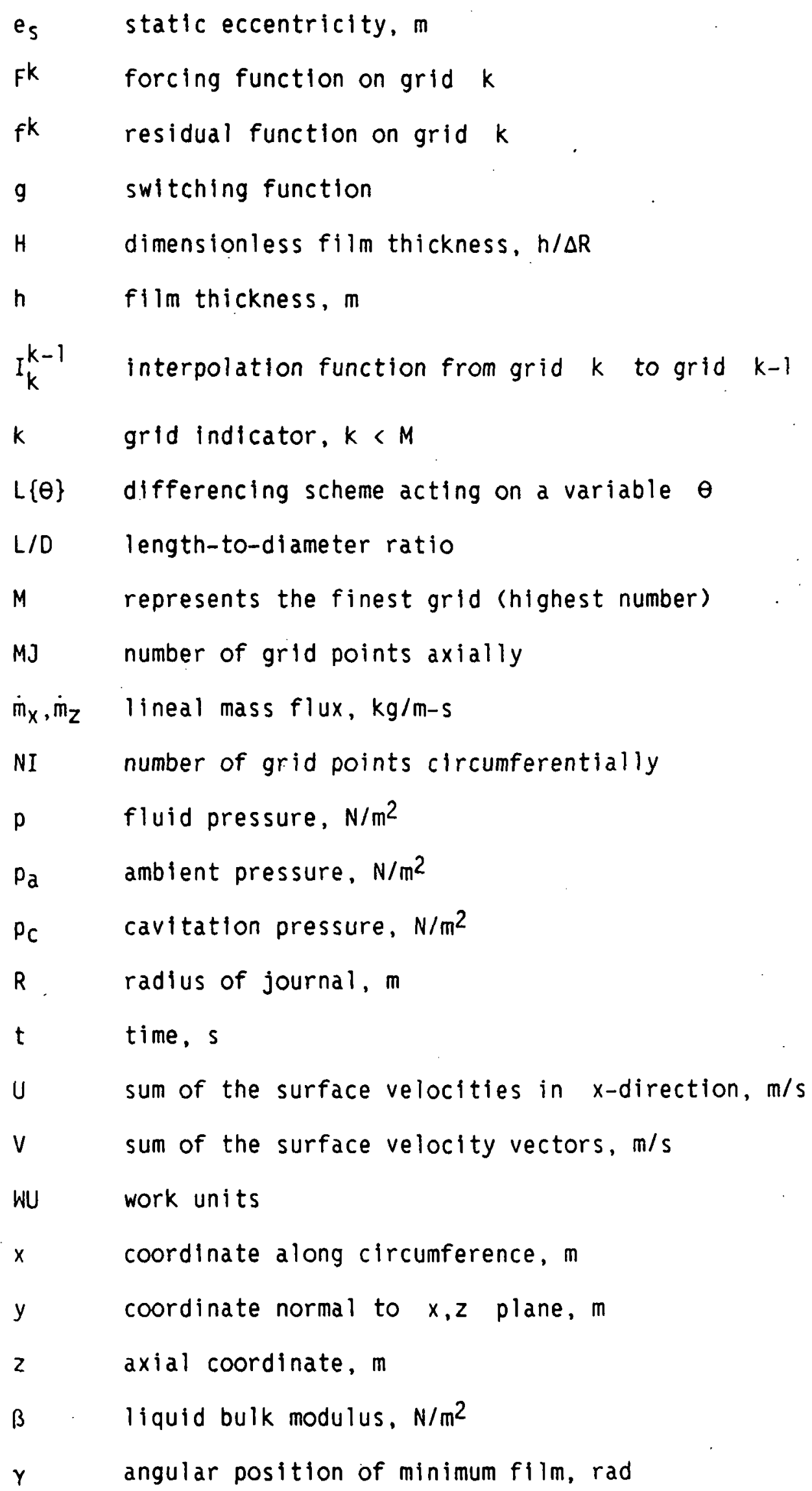




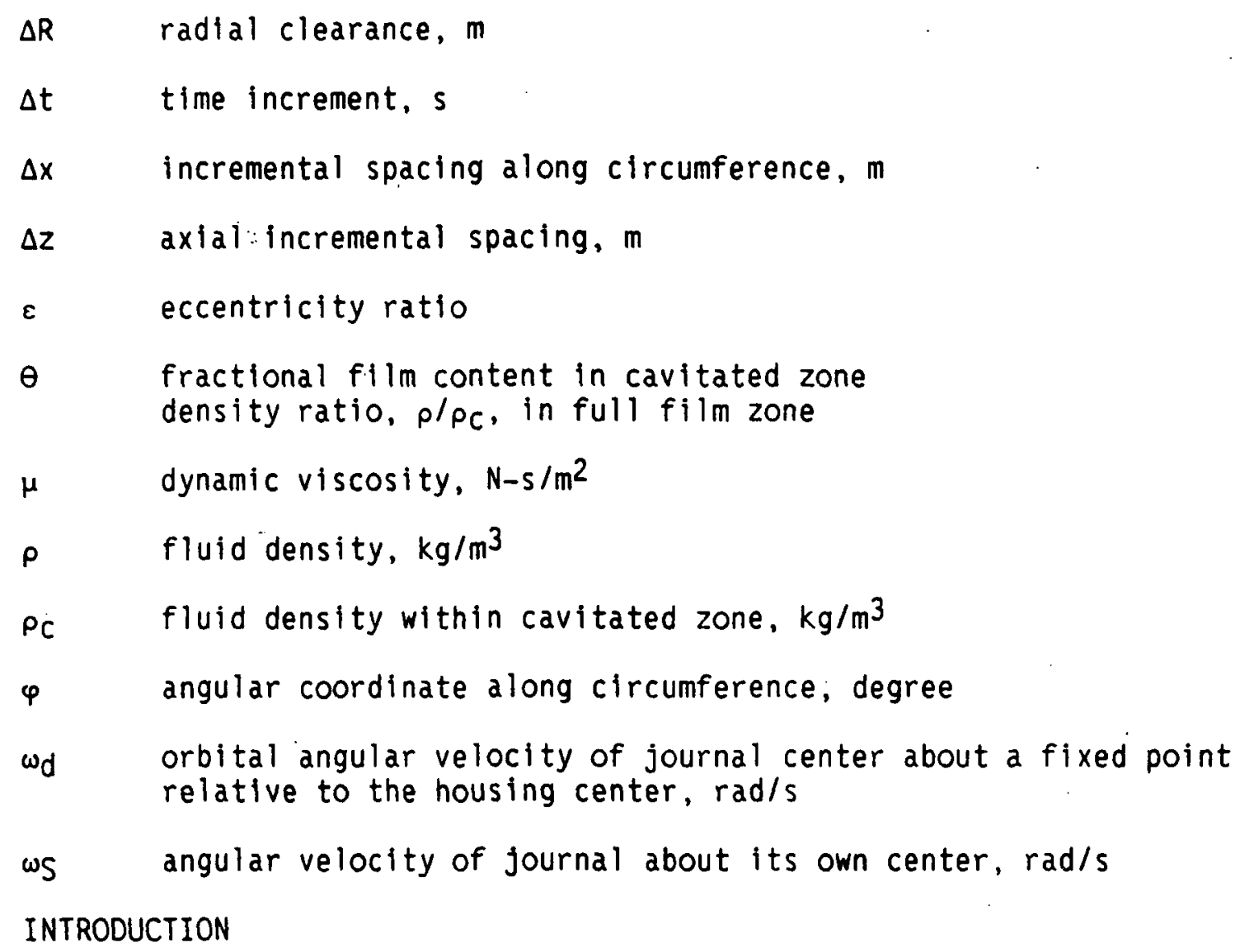

The presence of vapor cavitation in dynamically loaded journal bearings has become a topic of increasing importance. The use of increased loads and more complicated loading cycles has resulted in an increase in the occurrence of cavitation erosion problems. Examples of journal bearing applications include main and crankshaft bearings in diesel engines and a varlety of bearings in the aircraft industry, Dowson and Taylor (1). Dynamic loading can also lead to instablilities in the motion, such as whirling or whipping motion, which may damage the bearing. In order to avoid bearing damage, it is useful to predict the conditions under which the bearing will remain stable. The determination of these stability maps requires a knowledge of the hydrodynamic force terms. 
Previous static loading models, such as those using Swift-Stieber or Gümbel boundary conditions, assume a stationary cavitation bubble and are inadequate for high speed, dynamic applications. Under dynamic loading, changes in the local film thickness cause the bubble to grow, move downstream from the minimum film position, and collapse, Brewe (ㄹ).

A film model which effectively deals with dynamic loading has been formulated by Jakobsson-Floberg ( $\underline{3}$ ) and 01 sson (4). Besides accounting for a moving boundary, it also accommodates the flow within the cavitated region, manifested via a smeared mass or liquid striations. These striations have been observed in past experimental work. Because the theory assumes a zero pressure gradient within the cavitated zone, this mass flow is a Couette flow. The JFO theory accounts for both film rupture and film reformation, another advantage over previous methods. Unfortunately, the complexity of the JFO theory makes it difficult to apply (2) . Elrod and Adams $(\underline{5}, \underline{6})$ have developed an algorithm which automatically conforms to the JFO theory while being much simpler to code. It utilizes a switching function which eliminates the pressure gradient terms from the Reynold's lubrication equation at cavitated points.

A solution to the Elrod algorithm for a dynamically loaded problem has been formulated by Brewe (2). This direct solution to the finite differenced equations, i.e., no iterations required, utilizes an alternating direction implicit (ADI) scheme for the time march. When compared to a nonconservative film model (pseudo-Gümbel boundary conditions), as much as a 20-percent difference in load capacity is observed. Brewe's results agree excellently with the experimental work of Jakobsson and Floberg ( $\underline{3}$ ) for stationary cavitation. The present experimental data on nonstationary cavitation is limited, but Brewe's results compare reasonably well with the experimental 
work of Jacobson and Hamrock, $(\underline{7}, \underline{8})$. Unfortunately, Brewe's direct method requires two to three times the computational work needed by the nonconservative solution. The practical use of the Elrod algorithm in solving dynamic loading problems in industry requires the development of a more efficient computer solution.

Iterative techniques are not considered among the fastest methods of computer solution. However, newly developed techniques using multiple grid sizes have shown that it is possible to greatly reduce computational time. It is the purpose of this work to implement the multigrid technique developed by Achi Brandt, $(\underline{9}, \underline{10})$, in the iterative solution of the Elrod algorithm under dynamic loading conditions. As a point of reference, the multigrid method has also recently been implemented in an EHD lubrication problem by Lubrecht, ten Napel, and Bosma, (11).

The nature of the problem, i.e., the presence of discontinuous coefficients in the cavitated region, poses interesting difficulties in the application of the multigrid method. Therefore, one major objective is to find methods of making the multigrid technique as effective over the cavitated area as it is over areas of full film.

DESCRIPTION OF THE MODEL PROBLEM

The bearing motion consists of a journal undergoing a constant rpm as well as a noncentered circular whirl inside of a $360^{\circ}$ cylindrical bearing. The journal center moves through a prescribed dynamic cycle, (Fig. 1) from a minimum through a maximum eccentricity (see Table I for operating conditions).

The theoretical model assumes conditions of heavy loading, i.e., load carrying capacity $\gg$ surface tension forces in the liquid. An oil lubricant is used for which the vapor pressure is very nearly zero. It is assumed that the lubricant has been degassed and that only vapor cavitation is present. In actual experimental work with submerged bearings, this is a necessary 
condition, since gaseous cavitation forms at near ambient pressure and might prevent the occurrence of subamblent pressures. The flow balance into and out of the bearing cannot be maintained in the absence of subambient pressures. Within the cavitated region, a zero pressure gradient is assumed. In order to determine the load carrying capacity of the bearing under the prescribed motion, the flow equations are solved for the pressure variable. It is more convenient, however, to introduce another dependent variable, $\theta$, which has a dual interpretation regarding the full film region and the cavitated region. In the full film $(\theta \geq 1.0), \theta$ is the ratio $\rho / \rho_{C}$, which represents the ratio of film mass content to the mass content that would exist at the cavitation pressure $p_{C}$. In the cavitated region $(\theta<1.0), \theta$ represents the mass content that exists in the form of liquid striations. Pressure and density are related through the bulk modulus, $\beta$, such that $(\rho \partial p / \partial \rho)=\beta$. A switching function $(g(\theta))$ automatically eliminates the pressure term at cavitated points of the flow. That is,

$$
\begin{array}{lll}
\text { Uncavitated point } & (\theta \geq 1.0): & g=1 \\
\text { Cavitated point } & (\theta<1.0): & g=0
\end{array}
$$

THE GOVERNING EQUATION AND DIFFERENCING SCHEME

The Reynold's lubrication equation written in terms of the fractional film content, the switch function, and the bulk modulus becomes

$$
\frac{\partial(\theta h)}{\partial t}+\left(\frac{\vec{V}}{2}\right) \cdot \vec{\nabla}(\theta h)=\vec{\nabla} \cdot\left(\frac{\beta h^{3}}{12 \mu}\right) g(\theta) \vec{\nabla} \theta
$$

Note that the right-hand side, the pressure induced flow, completely disappears in the cavitated region where the switch function becomes zero. The finite difference equations are obtained using a control volume approach as was used by Brewe (2). A control volume (Fig. 2) is constructed about each nodal point and the net change in mass flow into the cell $\left(\dot{m}_{x+\Delta x / 2}-\dot{m}_{x-\Delta x / 2}\right)$ is 
equated to the total increase of mass $\left(\rho_{c} \theta h\right)$ in the cell over a time $\Delta t$. The control volume equivalent to the mass conservation equation is

$$
\rho_{c}\left(\frac{\partial(\theta h)}{\partial t}\right)=\frac{\Delta \dot{m}_{x}}{\Delta x}+\frac{\Delta \dot{m}_{z}}{\Delta z}
$$

In the full film region (all $g=1$ ), both the convective and pressure terms are central differenced, appropriate for the parabolic system. In the fully cavitated region (all $g=0$ ), the pressure terms are eliminated and the convective terms are upwind differenced, $\theta$ accounting for the mass transport. The combination of switching terms at the cavitation boundary automatically sets well posed boundary conditions between the two systems. The resulting $\Delta \dot{m}$ terms from the control volume analysis are

$$
\begin{array}{r}
\left(\Delta \dot{m}_{x}\right)_{\text {conv }}=\rho_{c}\left(\frac{U}{2}\right)\left\{h_{-1}\left(1-g_{-1}\right) \theta_{-1}-h_{0}\left(1-g_{0}\right) \theta_{0}+\frac{1}{2}\left[g_{-1} h_{-1}\left(2-g_{0}\right)\right.\right. \\
\left.\left.+g_{0} h_{0}\left(g_{-1}-2+g_{1}\right)-g_{1} h_{1} g_{0}\right]\right\} \\
\left(\Delta_{x}\right)_{\text {press }}=\left(\frac{\rho_{c}^{\beta}}{12 \mu}\right)\left(\frac{1}{\Delta x}\right)\left\{h_{-1 / 2}^{3} g_{-1}\left(\theta_{-1}-1\right)-\left(h_{-1 / 2}^{3}+h_{1 / 2}^{3}\right) g_{0}\left(\theta_{0}-1\right)\right. \\
\left.+\left[h_{1 / 2}^{3} g_{1}\left(\theta_{1}-1\right)\right]\right\}
\end{array}
$$

An Euler implicit time differencing scheme is used for stability purposes, giving:

$$
\frac{\theta h-\theta^{\star} h^{\star}}{\Delta t}=\frac{\Delta \dot{m}_{x}}{\rho_{c} \Delta x}+\frac{\Delta \dot{m}_{z}}{\rho_{c} \Delta z}
$$

where $\theta^{\star} h^{\star}$ signifies time $t-1$.

It should be noted that all terms on the right-hand side of this equation are evaluated at time $t$ and are therefore unknown. Along the axial boundaries, 1.e., along the edge of the bearing, the boundary condition is that of atmospheric pressure. The circumferential direction has wrap around 
boundary conditions. The problem and boundary conditions are described in more detail in (2).

THE MULTIGRID METHOD

Analysis of the Single Grid and the Residual Function

The iterative solution of a set of equations on a single grid generally has rapid convergence over the first few sweeps, but very slow convergence over most of the process. By examining the solution process, the reasons for this become clear. Assume the following continuous differential equation, $L\{\theta(x)\}=F(x)$; with suitable boundary conditions

for which the discretized set of equations on one grid take the form

$$
L^{k}\left\{\theta^{k}\right\}=F^{k}
$$

In the present notation, $k$ represents the particular grid size used, $L^{k}$ represents the differencing scheme acting on $\theta$, and $\theta$ is the exact solution of the differenced equations.

Let $\tilde{\theta}$ be the present approximation to the exact solution.. $\theta$. By substituting $\tilde{\theta}$ into the differenced equations, the following is obtained.

$$
f^{k}=F^{k}-L^{k}\left\{\tilde{\theta}^{k}\right\}
$$

where $f^{k}$ is referred to as the "residual function." The residual function is a means of analyzing the error left in the present approximation.

A Fourier analysis of $f^{k}$ breaks the error into its high-and lowfrequency components. High frequency is defined as wavelength less than or equal to four times the grid spacing. After a few relaxation sweeps, e.g., Gauss-Seidel, these high-frequency components are smoothed out, due to the fact that they are locally corrected. Once the error is all low frequency, the smoothing rate drops drastically. The grid spacing is too fine to efficiently smooth these low-frequency terms Brandt ( $\underline{9})$. 


\section{The Roles of High- and Low-Frequency Error Smoothing}

The basic thrust of multigrid is to utilize coarser grids to handle these low-frequency error terms. As long as the residual function, i.e., the error, is well represented on a particular grid, that grid can quickly smooth its own "high-frequency" terms and send an appropriate correction back to the finer grid. By utilizing coarser and coarser grids, all of the low frequency terms are treated similarly. Besides their ability to efficiently deal with lowfrequency terms, coarse grids also have fewer nodal points to sweep through, making the coarse grid sweeps very cheap.

Whereas moving to coarser grids smooths the low-frequency error, fine grid updates during the multigrid process have value in improving the accuracy of the present solution. The role of relaxation on the finer grid is to resolve its own high-frequency components as well as smooth the high-frequency error which is produced by interpolation from the coarser grid.

\section{Coarse Grid Representation}

The fine grid problem itself, i.e., $L^{k}\left\{\tilde{\theta}^{k}\right\}=F^{k}$, is not what is really being represented on the coarse grid $k-1$. The actual purpose of the coarse grid is to solve for a correction vaiue, $\theta_{c}^{k-1}$

$$
\theta_{c}^{k-1}=\theta^{k-1}-\tilde{\theta}^{k-1}
$$

as a function of the amount of residual error that is left in the present approximation on the fine grid, i.e., $f^{k}$.

For a nonlinear problem, the full approximation storage (FAS) mode must be used. This method stores the entire value of $\tilde{\theta}^{k-1}$ on the coarse grid $k-1$ instead of just the correction value $\theta_{c}^{k-1}$. If used on a linear problem, the 
equations reduce to those for the linear mode Brandt $(9)$. The existence of nonlinearities in the convective mass flow contribution necessitates the use of the FAS mode. Using FAS, the coarse grid problem becomes

$$
\tilde{\theta}_{\text {Initial }}^{k-1}=I_{k}^{k-1}\left(\tilde{\theta}^{k}\right)
$$

where $I_{k}^{k-1}$ is the interpolation operator from the fine to the coarse grid. Also,

$$
F^{k-1}=I_{k}^{k-1}\left(F^{k}-L^{k} \tilde{\theta}^{k}\right)+L^{k-1}\left\{\tilde{\theta}^{k-1}\right\}
$$

Solve

$$
\begin{gathered}
L^{k-1}\left\{\tilde{\theta}^{k-1}\right\}=F^{k-1} \\
\theta_{c}^{k-1}=\tilde{\theta}^{k-1}-\tilde{\theta}_{\text {Initial }}^{k-1}
\end{gathered}
$$

Using the interpolation operator $I_{k-1}^{k}$, i.e., from the coarse to the fine grid, we obtain

$$
\tilde{\theta}_{\text {new }}^{k}=\tilde{\theta}_{\text {old }}^{k}+I_{k-1}^{k}\left(\theta_{c}^{k-1}\right)
$$

The fact that the coarse grid is solving for $\theta_{c}^{k-1}$ as a function of $f^{k}$ has two important consequences. The first is that $f^{k}$ must be well represented on the coarse grid in order that $\theta_{c}^{k-1}$ is an accurate correction value. Therefore, care must be taken that $f^{k}$ is well smoothed on the fine grid before transferring it to the coarse grid.

The second consequence is that. it is not necessary that the coarse grid differencing scheme, $L^{k-1}\left\{\tilde{\theta}^{k-1}\right\}$, exactly match the fine grid differencing scheme, $L^{k}\left\{\tilde{\theta}^{k}\right\}$. This can be seen from the following consideration, $\mathfrak{i} . e .$, the concept of solving for a correction value on the coarse grid arises because

$$
\left[L^{k-1}\left\{\theta^{k-1}\right\}-L^{k-1}\left\{\tilde{\theta}^{k-1}\right\}\right] \begin{gathered}
\text { should } \\
\text { represent }
\end{gathered}\left[L^{k}\left\{\theta^{k}\right\}-L^{k}\left\{\tilde{\theta}^{k}\right\}\right] .
$$


It can be shown that the multigrid equations follow from this basic concept. Now considering the RHS, $\theta^{k}$ is unknown, although we can use the fact that $L^{k}\left\{\theta^{k}\right\} \equiv F^{k}$. Therefore,

$$
\text { RHS }=\left[F^{k}-L^{k}\left\{\theta^{k}\right\}\right]=f^{k}=\text { Residual function. }
$$

From the definition of $\tilde{\theta}_{C}^{k-1}$, and the fact that $L^{k-1}$ is a linear operator, the LHS can be written

$$
L^{k-1}\left\{\theta_{c}^{k-1}\right\}=L^{k-1}\left\{\theta^{k-1}\right\}-L^{k-1}\left\{\tilde{\theta}^{k-1}\right\}
$$

The full equation then becomes the standard linear multigrid equation

$$
L^{k-1}\left\{\theta_{c}^{k-1}\right\}=I_{k}^{k-1}\left(f^{k}\right)
$$

which can then be adapted to the nonlinear form expressed in Eq. (3).

Therefore, the terms which must closely represent each other are the bracketed terms, not the individual components within. Thus there is some flexibility in creating $L^{k-1}\left\{\tilde{\theta}^{k-1}\right\}$. Especially if the problem contains rapidly changing spatial coefficients, the coarse grid differencing scheme will have to be a modified version of the fine grid scheme.

Multigrid Cycle

Various multigrid cycles can be used. When developing a multigrid code, it is best to use a prescribed cycle so that the results obtained by testing different relaxation and interpolation schemes can be easily compared. One example of this type is the V-cycle. One V-cycle consists of the following: a predefined number of sweeps on each grid in descending order of fineness until the coarsest grid is reached; iterating the coarsest grid problem to convergence; and a predefined number of sweeps on each grid in ascending order. The goal in multigrid is to obtain an order of magnitude error reduction per cycle. Once the best relaxation and interpolation schemes for the problem have 
been determined, the adaptive multigrid method can be used. This is generally more efficient than the prescribed cycle method. The adaptive algorithm stays on a particular grid until convergence on that grid has slowed to a defined "slow" rate, at which time it automatically moves to the next coarser grid. Whenever it converges to the set tolerance at a particular grid, it moves back up to the next finer grid:

\section{Determination of Smoothness Between Grids}

The process of determining smoothness between grids is described using FAS and the adaptive multigrid algorithm. Let $M$ denote the finest grid.

$$
L^{M}\left\{\tilde{\theta}^{M}\right\}=F^{M}
$$

After each relaxation on grid $M$, the program decides whether to stay on the present grid or move to a coarser one. For simplicity sake, this is often done by measuring the rate of convergence and determining a cutoff rate, i.e., if convergence is "slow," the program will move to a coarser grid (see Fig. 3(a) for a schematic of the general problem). This method infers that slow convergence signifies a smoothed residual function. It assumes that the presence of high-frequency terms will show up in a rapidly decreasing global error term, where

$$
\text { global error }=\sqrt{\sum_{i, j}\left(\tilde{\theta}_{i, j_{0 l d}}-\tilde{\theta}_{i, j}\right)^{2}}
$$

This is not a bad assumption if there are no local areas containing rapidly changing spatial coefficlents. Problems may occur if local regions of high-frequency residuals exist within a globally smooth domain. The global error term may not be affected by the high-frequency errors and will interpolate the solution to the coarser grid, where the residual error of the local region will not be well represented. Some other method of determining 
the smoothness of this local area is needed in such a case. Additional sweeps over the fine grid, or parts of the fine grid, are then needed to smooth the error locally.

Assuming that the residuals have been sufficiently smoothed on the fine grid $k$, the problem moves to grid $k-1$ and relaxes Eq. (3). The program either returns to the fine grid if the solution is converged, or goes to a coarser grid if the convergence is slow and the residuals are smooth. When the coarsest grid is reached, a converged solution is obtained by continued relaxation or by direct solution. When a converged solution is obtained on a grid $k-1$, the solution is updated on grid $k$ using Eq. (4). APPLICATION OF MULTIGRID

The use of implicit time differencing necessitates the solution of an NIXMJ set of equations at each time step. In this study, no attempt is made to use multigrid across physical time. Multigrid is used to facilitate the iterative solution of the NIXMJ set of equations within each time step.

The fine grid (M) equations take the form $L^{M}\left\{\theta^{M}\right\}=F M$, where $L^{M}\left\{\theta^{M}\right\}$ represents the differencing scheme of $\theta$ described earlier. The forcing function, $F M$, represents terms from the previous time step which evolve from the implicit time differencing scheme.

$$
F^{M}=\frac{\theta^{\star} h^{\star}}{\Delta t}
$$

The coarse grid representation as derived above are used to implement the multigrid procedure. A flow schematic of the procedure used is shown in Fig. $3(b)$.

Full weighting is used for the fine-to-coarse grid interpolations, taking into account all nine fine grid points associated with the coarse grid equivalent point, 


$$
\begin{aligned}
\theta^{k-1}=I_{k}^{k-1} \theta^{k}=\left(\frac{1}{16}\right)\left[\theta_{i-1, j-1}^{k}+2 \theta_{i-1, j}^{k}\right. & +\theta_{i-1, j+1}^{k}+2 \theta_{i, j-1}^{k}+4 \theta_{i, j}^{k} \\
& \left.+2 \theta_{i, j+1}^{k}+\theta_{i+1, j-1}^{k}+2 \theta_{i+1, j}^{k}+\theta_{i+1, j+1}^{k}\right]
\end{aligned}
$$

Both linear and third degree polynomial interpolation from coarse to fine grid were tested, and several different relaxation schemes were tried.

The Switch Function

The values of the switching function, $g(\theta)$, are not allowed to change during a multigrid solution process. Attempts to let them vary with the solution led to major instabilities. The $g$ values at the new time step, $g^{t}$,

are first determined from the previous solution. i.e., $\theta_{\text {conv }}^{t-1}$. Using these values at time step $t$, the fine grid equations are relaxed a certain number of times, after which the $g$ values are updated to the present $\theta_{\text {approx }}^{t}$. These $g$ values are then used throughout the multigrid solution. RESULTS

Excellent results were obtained over the time steps prior to the start of cavitation, both in terms of comparison with a single grid iterative solution and comparison with Brewe's direct solution. These results are summarized in Table II.

Comparisons with single grid iteration are done on the basis of work units (WU) used, where 1 WU is equivalent to I relaxation sweep over the finest grid. Letting $M$, i.e., total number of grids, represent the finest grid and $k a$ coarser grid, numbered in decreasing order respectively, the equivalent wU used by grid $k$ is

$$
W U^{k}=4^{(k-M)}
$$

The following results were obtained for the test case having a maximum eccentricity of 0.8 and a minimum eccentricity of 0.1 (see Table I). This is 
one of the more difficult cases to run, since very high pressures and large pressure gradients are induced near the minimum film thickness. The pressure gradients and the bubble shape change relatively rapidly with time.

In all of the trials, a system of three grids was used. The addition of a fourth coarsest grid had a negligible effect. For comparison purposes, a single grid solution using Gauss-Seidel relaxation on a 96- by 24-point mesh was run ( 96 points circumferentlally and 24 points axially). The 96 - by 24-point mesh ensures a grid aspect ratio $(A R=\Delta x / \Delta z)$ of 1 . It required about 300 WU per time step.

Gauss-Seidel (G-S) and Jacobi (J) relaxation schemes with no overrelaxation were found to be the most effective smoothers for this problem. Circumferential line relaxation, i.e., solving simultaneously each line of points in the circumferential direction, is an effective smoother, but is not worth the substantially greater computational time needed to solve for the periodic boundary conditions, which introduce corner terms to the tridiagonal matrix. Line relaxation is used as a local smoother, however, when cavitation develops. Both the G-S and $J$ relax points in the direction of the flow, i.e., the circumferential direction, sweeping across the axial direction. Sweeping across the circumferential direction is not very effective, nor is a combination of axial and circumferential sweeps. A red-black scheme is also not very effective.

The difference between the G-S and the $J$ schemes when used in the multigrid process is extremely small. J relaxation uses an average of $0.5 \mathrm{WU}$ more than G-S per time step. The reason seems to be that the $J$ multigrid uses the same number of fine and medium grid sweeps as does the G-S per solution and makes up for its lower efficiency by using a greater number of the coarsest grid sweeps, which are very cheap. The advantage of using $J$ 
when working with a parallel processing computer is that the inner loop is vectorizable, since all points in a line are substituted at the end of the line instead of point by point. Using the CRAY XMP, one $J$ sweep takes one-tenth the CPU time of a G-S sweep.

A multigrid solution using G-S relaxation along the direction of flow and a linear interpolation scheme from the coarse to fine grid was first tested. The solution on a 48 - by 48 -point fine mesh $(A R \cong 3.1$ ) required an average of 24 WU per time step. The solution on the 96 - by 24-point mesh required an average of 14 WU per time step, which is nearly 22 times faster than the single grid solution.

A third degree polynomial interpolation scheme from coarse to fine grid was also tested. Using the same $96-$ by 24 -point mesh and $G-S$, this scheme reduces the work per time step to an average of $7.5 \mathrm{WU}$, half the work used by the linear scheme. Also, the third degree polynomial routine takes virtually the same amount of CPU time as the linear routine on the CRAY XMP, making it highly worthwhile. This scheme used approximately 1/40 of the work used by single grid iteration.

The adaptive multigrid cycle was used to obtain the above results. To determine the efficiency, however, a $V$-cycle was also run. Each cycle reduces the error by nearly an order of magnitude.

The results also compare well with Brewe's direct numerical solution, both in accuracy and CPU time. Also, load capacities were compared at various time steps. The greatest difference found between the load capacity values is two parts in 10000 . Both the direct and the multigrid codes are vectorized to the highest efficiency. Both were run on the CRAY XMP for 5000 time steps of uncavitated flow. The direct solution took $1086 \mathrm{sec}$ CPU, while the multigrid code took $57 \mathrm{sec}$ CPU, about $1 / 20$ the CPU time of the direct solution. 
The presence of cavitated points in the flow, i.e., the presence of an area having $g=0$ bounded by points having $g=1$, requires a more involved approach. On a single grid, the algorithm handles the cavitation as efficiently as it would an uncavitated configuration. Problems begin to occur when coarser grids are added.

Initially, the coarse grid cavitation area was determined by injecting corresponding fine grid g's directly to the coarse grid points. Figure 4 shows graphs of the residual function at similar states in the solution for both an uncavitated and a cavitated configuration using this scheme. These graphs were obtained with no extra smoothing around the cavitated area. As can be seen, high-frequency local-error terms exist around the cavitated boundary, whereas the uncavitated region has already been well smoothed. If the program is allowed to continue from this point, it moves to the coarser grid, where the cavitated boundary residuals are not well represented. Depending on how unsmooth the boundary residuals are, $V$-cycle results range from 40 -percent error reduction per cycle to a slight divergence of error terms per cycle.

Extra local smoothing helps immensely, as would be expected. The best results are obtalned by using a local circumferential line relaxation scheme over the cavitated region and boundary points. This scheme is a very powerful smoother and is also expedient, since, as a local smoother, it reduces to a purely tridiagonal matrix of a relatively small number of points.

The problem still remains of deciding how many local smoothing sweeps is "enough," or whether any are necessary at all. If the number of sweeps is set such that the most difficult cavitation configurations converge efficiently, then configurations having smoother initial residuals become much less efficient. Some type of smoothness indicator is necessary. The present 
routine takes the fine grid residual function, $f^{k}$, and interpolates it down to grid $k-1$ using the same full weighting as in an actual grid switch. The coarse grid values are then interpolated linearly back up to the fine grid $k$. These then represent "smooth" fine grid residuals and are compared with the actual residuals. If any of the actual terms fall outside of an envelope placed around the smooth term, the problem is deemed unsmooth and local smoothing is done.

The above procedure does much to stabilize the solution process, resulting in an average usage of 40 WU per time step solution. For the g-injection model, however, order of magnitude error reduction per cycle is not usually obtained, and certain cavitation configurations do occur which are very difficult or impossible to solve.

As mentioned earlier, the coarse-grid operator $L^{K-1}$ need not be the same as the fine-grid operator $L^{M}$. Because of this fact, some latitude in handling the values of the $g$ coefficient in the coarse grid equations is permissible. This led to a coarse-grid determination scheme for the $g$ values that not only circumvented occurrences of instability due to injection but had a major beneficial effect on the. solution. Recall, the $g$ values on the finest grid are determined by the value of $\theta$ at each nodal point, i.e., g has a value of 1 at full film points and a value of 0 at cavitated points. It was found that stability of the solution across all possible cavitation configurations can be obtained by defining a parameter FG as:

$$
\begin{gathered}
F G=\left[g_{i-1, j-1}^{k}+g_{i-1, j}^{k}+g_{i-1, j+1}^{k}+g_{i, j-1}^{k}+g_{i, j}^{k}+g_{i, j+1}^{k}+g_{j+1, j-1}^{k}\right. \\
\left.+g_{i+1, j}^{k}+g_{i+1, j+1}^{k}\right] \\
\text { If } F G=0 ; \quad g^{k-1}=0 \\
\text { If } F G \neq 0 ; \quad g^{k-1}=1
\end{gathered}
$$


In other words, a fine grid point must have a $g^{k}$ value of 0 and must be surrounded (all eight points) by points having $g^{k}=0$ in order for the corresponding coarse grid point to be set to $g^{k-1}=0$. Other schemes were found to work but were not as efficient. This scheme resulted in an average of 20 WU per time step, the number of WU ranging from 11 to $35 \mathrm{WU}$. The solution process remains stable throughout bubble formation and bubble collapse. The time steps which required the most work units occurred at the very beginning of bubble formation, when there were very few cavitated points, and during bubble collapse. While the bubble is collapsing, it is also experiencing its greatest amount of movement downstream, so it might be this movement rather than the process of collapse which requires more solution time.

A V-cycle analysis shows that better than an order of magnitude error reduction per cycle is obtained, though at the cost of extra smoothing on the finer grids.

The results for cavitated flow also compare well with Brewe's direct solution. The same bubble shape, motion, and duration are obtained from both programs. Figure 5 contains computed pressure distributions at various time steps. The cavitated area is indicated by the flat area of zero pressure gradient. Comparison of load capacity terms shows a maximum difference of five parts in 10000 in the cavitated region. When run on the CRAY XMP, the direct solution of 5000 time steps of cavitated flow again takes $1086 \mathrm{sec}$ CPU. The multigrid solution of 5000 time steps of cavitated flow takes $150 \mathrm{sec}$ CPU, approximately one-eighth the CPU taken by the direct solution. Even though the cavitated configurations take more CPU than do the uncavitated configurations, the multigrid solution still represents a very significant savings over the direct method. 
In his paper on the direct ADI solution of the Elrod algorithm, Brewe (2) states that his solution uses two to three times the computational time used by an iterative solution of a nonconservative film model using pseudo-Gümbel boundary conditions. This nonconservative film model is only suited to steadystate conditions, but is often used by industry. Thus, the multigrid solution of the Elrod algorithm requires about one-tenth to one-third the computational time (for uncavitated and cavitated flow respectively) of the nonconservative film model solution, while still retaining the more realistic representation of the flow.

CONCLUSION

A multigrid iterative technique is used in the solution of the Elrod algorithm for the case of a dynamically loaded journal bearing undergoing cavitation. This solution is compared both to a single grid iterative solution in terms of work used, and to a direct ADI solution in terms of computer time required. Excellent results are obtained both prior to and during cavitation, although the presence of cavitation does introduce difficulties in the solution process.

The best results are obtained using the following: a grid aspect ratio of 1; full weighting interpolation from the fine grid to the coarse grid; third degree polynomial interpolation from the coarse grid to the fine grid; either Gauss-Seidel or Jacobi relaxation with no overrelaxation. Implementing these techniques, the solution at time steps prior to cavitation uses $1 / 40$ the amount of work used by a single grid iterative Gauss-Seidel solution and 1/20 the computer time used by the direct ADI solution. During cavitation, the multigrid solution uses $1 / 15$ the amount of work used by a single grid G-S solution and one-eighth the computation time used by the direct ADI solution. 
Based on the results stated in this paper, it is evident that the solution of the Elrod algorithm using multigrid techniques provides an extremely viable method to industry for the solution of journal bearing problems.

\section{REFERENCES}

1. Dowson, D. and Taylor, C.M., 1979, "Cavitation in Bearings," Annual Review of Fluid Mechanics, Vol. 11, M. VanDyke, J.V. Wehausen, and J.L. Lumley, eds., Annual Reviews Inc., Palo Alto, CA, pp. 35-66.

2. Brewe, D.E., 1986, "Theoretical Modeling of the Vapor Cavitation in Dynamically Loaded Journal Bearings," Journal of Tribology, Vol. 108, No. 4 , pp. 628-638.

3. Jakobsson, B., and Floberg, L., 1957, "The Finite Journal Bearing Considering Vaporization," Rept. No. 190, Chalmers University of Technology, Goteborg, Sweden.

4. Olsson, K.0., 1965, "Cavitation in Dynamically Loaded Journal Bearings," Rept. No. 308, Chalmers University of Technology, Goteborg, Sweden.

5. Elrod, H.G. and Adams, M.L., 1975, "A Computer Program for Cavitation and Starvation Problems," Cavitation and Related Phenomena in Lubrication, D. Dowson, M. Godet, and C.M. Taylor, eds., Mechanical Engineering Publications, New York, pp. 37-42.

6. Elrod, H.G., 1981, "A Cavitation Algorithm," Journal of Lubrication Iechnology, Vol. 103, No. 3, pp. 350-354.

7. Jacobson, B.O., and Hamrock. B.J., 1983, "Vapor Cavitation in Dynamically Loaded Journal Bearings," 2nd International Conference on Cavitation, Mechanical Engineering Publications, New York, pp. 133-140.

8. Jacobson, B.0., and Hamrock, B.J., 1983, "High-Speed Motion Picture Camera Experiments of Cavitation in Dynamically Loaded Journal Bearings, "Journal of Lubrication Technology, Vol. 105, No. 3, pp. 446-452. 
9. Brandt, A., 1977, "Multi-Level Adaptive Solutions to Boundary-Value Problems," Mathematics of Computation, Vol. 31, No. 138, pp. 333-390.

10. Brandt, A., 1984, "Multigrid Techniques: 1984 Guide; With Applications to Fluid Dynamics," Computational Fluid Dynamics, Vol. 1, VKI-LS-1984-04, Von Karman Institute for FLuid Dynamics, Rhode St. Genese, Belgium, 1984, Paper No. 1.

11. Lubrecht, A.A., Ten Napel, W.E., and Bosma, R., 1986, "Multigrid, An Alternative Method for Calculating Film Thickness and Pressure Profiles in Elastohydordynamically Lubricated Line Contacts," Journal of Tribology, Vol. 108, No. 4, pp. 551-556.

TABLE I. - OPERATING CONDITIONS

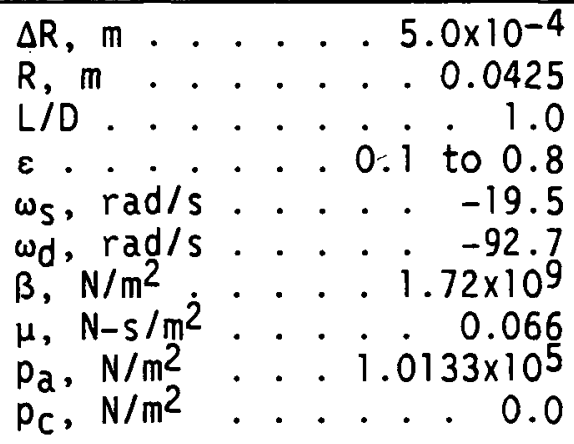


TABLE II. - RESULTS

(a) Prior to cavitation, work units ( $\left(U^{2}\right)$ used per time step solution.

\begin{tabular}{|c|c|c|c|c|}
\hline $\begin{array}{l}\text { Number } \\
\text { of } \\
\text { grids }\end{array}$ & $\begin{array}{l}\text { Aspect } \\
\text { ratio, } \\
\text { AR }\end{array}$ & $\begin{array}{l}\text { Type of } \\
\text { relaxation }\end{array}$ & $\begin{array}{l}\text { Type of } \\
\text { interpolation } \\
k \text { to } k+1\end{array}$ & $\begin{array}{c}\text { Average } \\
\text { WU }\end{array}$ \\
\hline $\begin{array}{l}1 \\
3 \\
1\end{array}$ & $\begin{array}{c}1 \\
3.1 \\
1 \\
1\end{array}$ & $\begin{array}{c}\text { G-S } \\
\downarrow \\
J\end{array}$ & $\begin{array}{l}\text { Linear } \\
\text { Linear } \\
\text { Third-degree } \\
\text { polynomial } \\
\text { Third-degree } \\
\text { polynomial }\end{array}$ & $\begin{array}{l}300 \\
24 \\
14 \\
7.5 \\
8.0\end{array}$ \\
\hline
\end{tabular}

(b) Prior to cavitation, CPU time used for solution of 500 time steps on Cray XMP.

\begin{tabular}{|c|c|}
\hline \multicolumn{1}{|c|}{ Type of solution } & $\begin{array}{l}\text { CPU } \\
\text { time, } \\
\text { sec }\end{array}$ \\
\hline Direct - ADI (96- by 24-point mesh) & 1086 \\
$\begin{array}{l}\text { Multigrid - three grids (Jacobi } \\
\text { relaxation, third-degree poly- } \\
\text { nomial interpolation from coarse } \\
\text { to fine, 96- by 24-point mesh) }\end{array}$ & 57 \\
\hline
\end{tabular}

(c) During cavitation

\begin{tabular}{|c|c|c|c|c|c|}
\hline $\begin{array}{c}\text { Number } \\
\text { of } \\
\text { grids }\end{array}$ & $\begin{array}{l}\text { Type of } \\
\text { solution }\end{array}$ & g-mode $1^{b}$ & $\begin{array}{l}\text { Average } \\
\text { WU per } \\
\text { time step } \\
\text { solution }\end{array}$ & $\begin{array}{l}\text { Cray CPU } \\
\text { time for } \\
5000 \text { time } \\
\text { steps, } \\
\text { sec }\end{array}$ & $\begin{array}{l}\text { Stability of } \\
\text { solution } \\
\text { process }\end{array}$ \\
\hline $\begin{array}{l}1 \\
3\end{array}$ & $\begin{array}{l}G-S \\
G-S \text { or } J \text {; } \\
\text { no } L C \\
G-S \text { or } J \\
\text { and } L \\
G-S \text { or } J \\
\text { and } L \\
G-S \text { or } J \\
\text { and } L \\
\text { Direct (ADI) } \\
\text { Nonconformal } \\
\text { film modi- } \\
\text { fication }\end{array}$ & $\begin{array}{l}g=f c n(\theta) \\
\text { Injection } \\
\text { Injection } \\
g l \\
\text { gsur } \\
g=f c n(\theta) \\
\text { Stationary } \\
\text { cavita- } \\
\text { tion area }\end{array}$ & $\begin{array}{c}300 \\
40 \\
40 \text { to } 50 \\
20 \\
---\end{array}$ & $\begin{array}{l}---- \\
--- \\
--- \\
150 \\
1086 \\
N 500\end{array}$ & $\begin{array}{l}\text { Stable } \\
\text { Unstable } \\
\text { Unstable } \\
\text { Stable }\end{array}$ \\
\hline \multicolumn{6}{|c|}{$\begin{array}{l}\text { bG-models: } \\
\text { Injection: } g^{M}=f\left(n(\theta)=1 \text { or } 0 ; g^{k<M}=\text { corresponding } g^{k+1} \text {. }\right. \\
\text { gl: } g^{M}=f\left(n(\theta) ; a l l \quad g^{k}<M=1 \text {. }\right. \\
\text { gsur: } g^{M}=f\left(n(\theta) ; g^{k<M}=0 \text { only if corresponding } g^{k+1}=0 \text { and is }\right. \\
\text { surrounded by } g^{k+1}=0 \text { points. }\end{array}$} \\
\hline
\end{tabular}



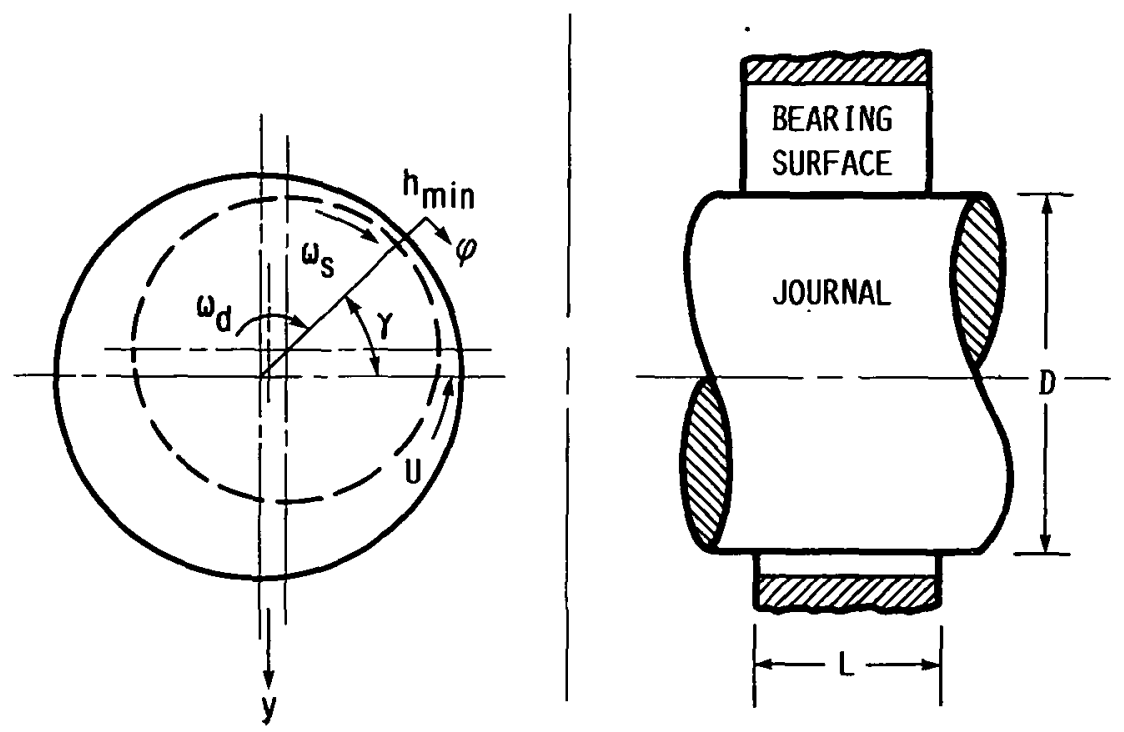

(A) JOURNAL BEARING CONFIGURATION.
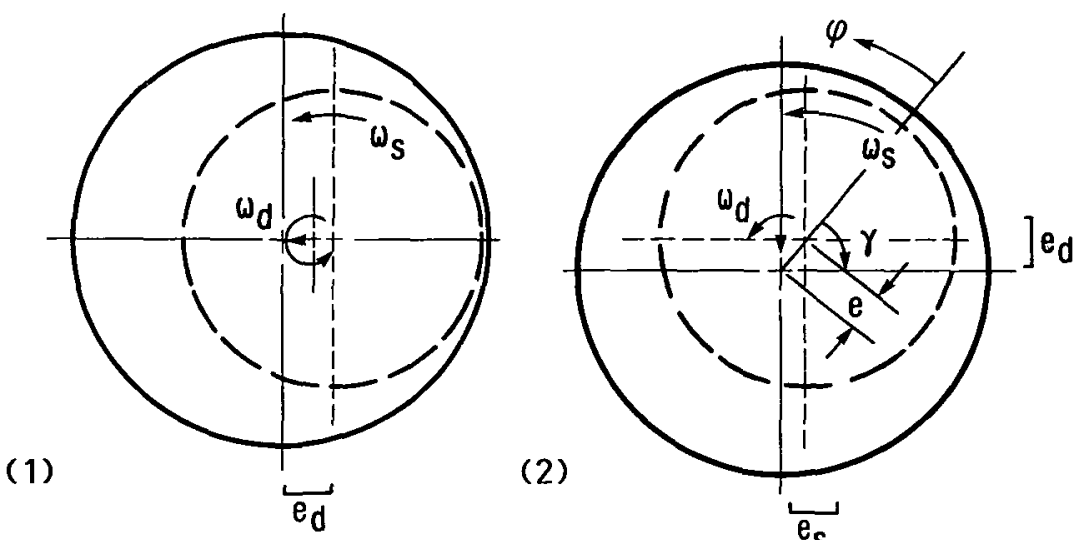

(3)
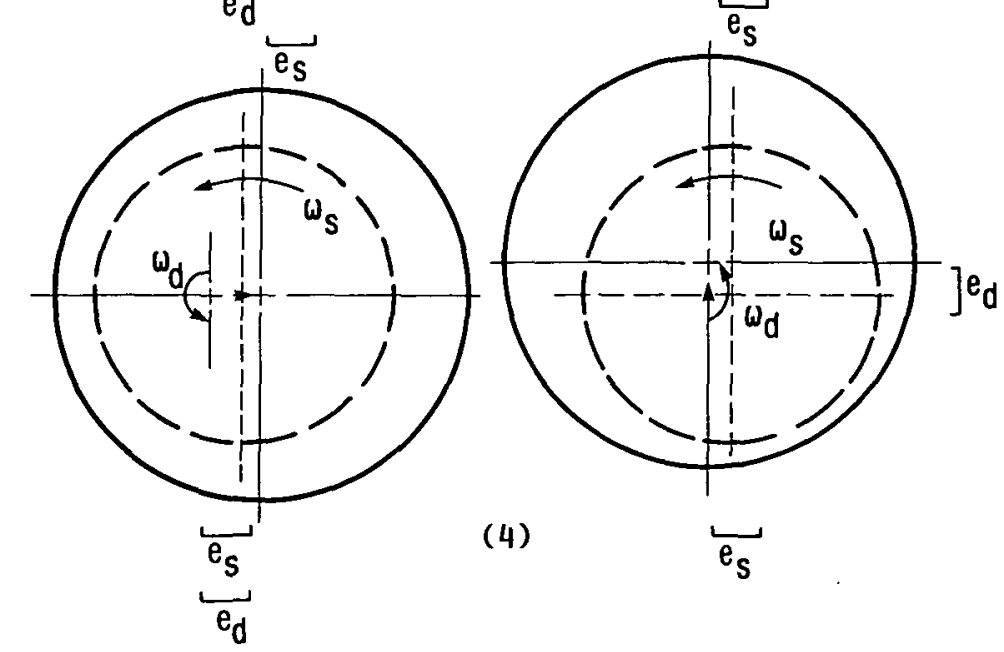

(B) BEARING GEOMETRIES AT FOUR DIFFERENT TIMES.

FIGURE 1. - JOURNAL BEARING CONFIGURATION. 


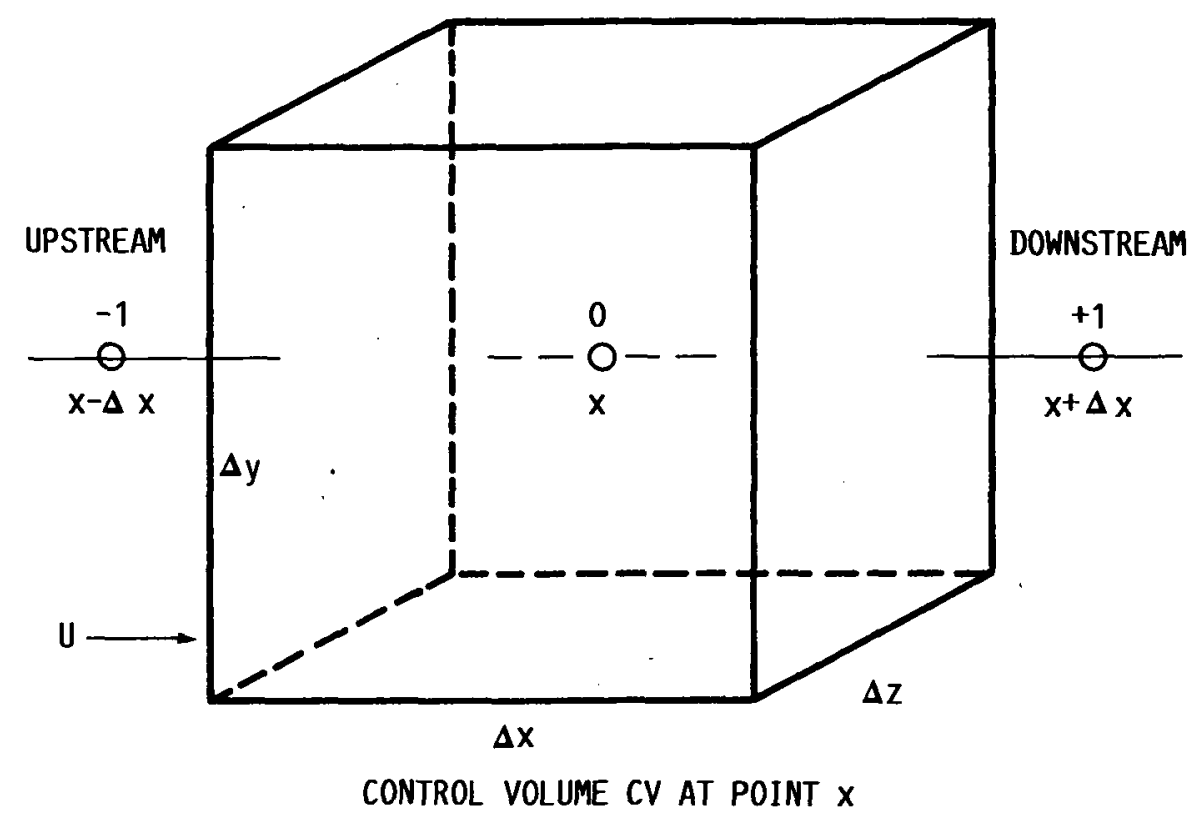

FIGURE 2. - CONTROL VOLUME APPROACH. 


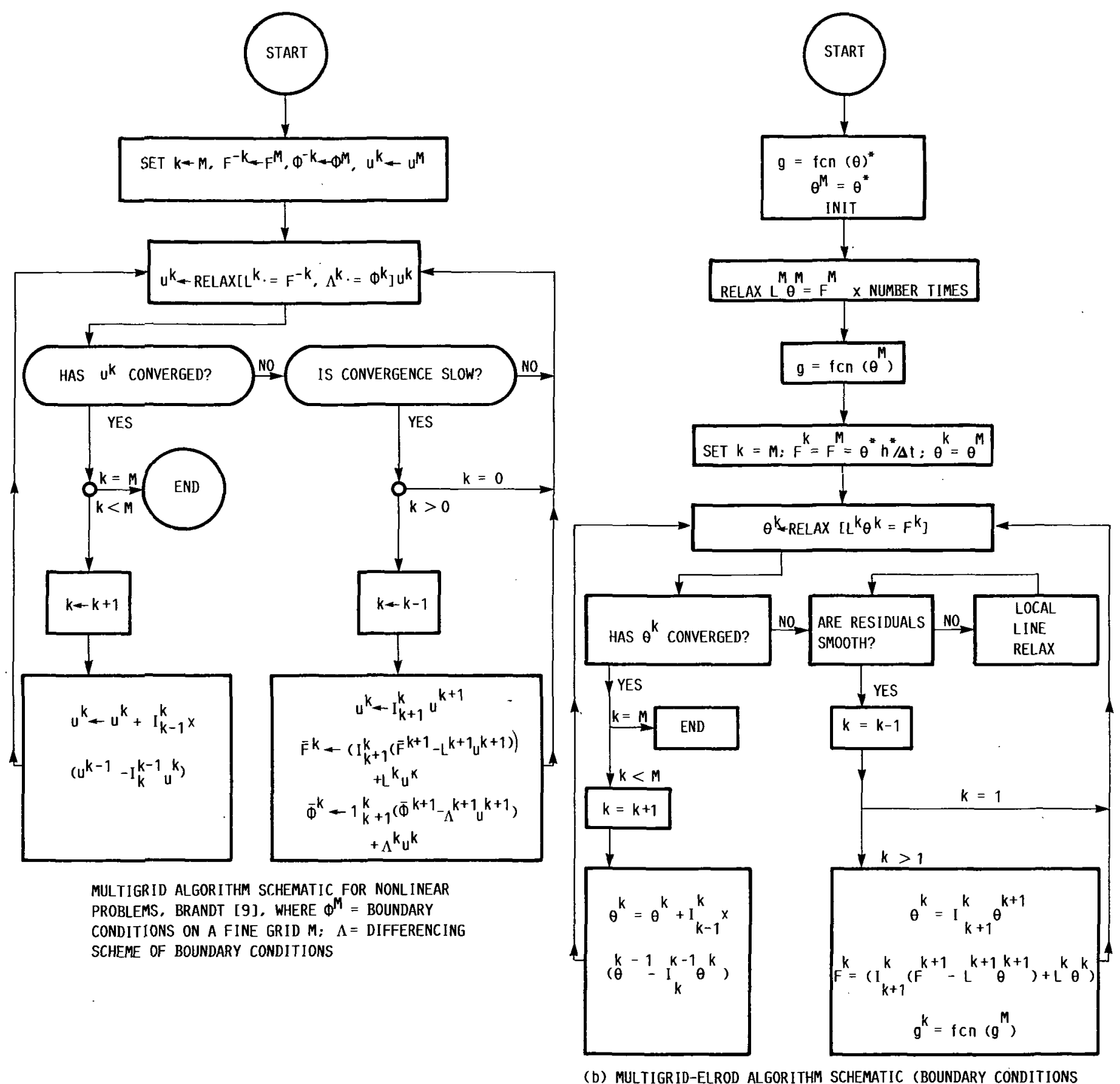

(b) MULTIGRID-ELROD ALGORITHM SCHEMATIC (BOUNDARY CONDITIONS NOT INCLUDED).

FIGURE 3. - MULTIGRID ALGORITHM SCHEMATICS WITH FULL APPROXIMATION STORAGE. 


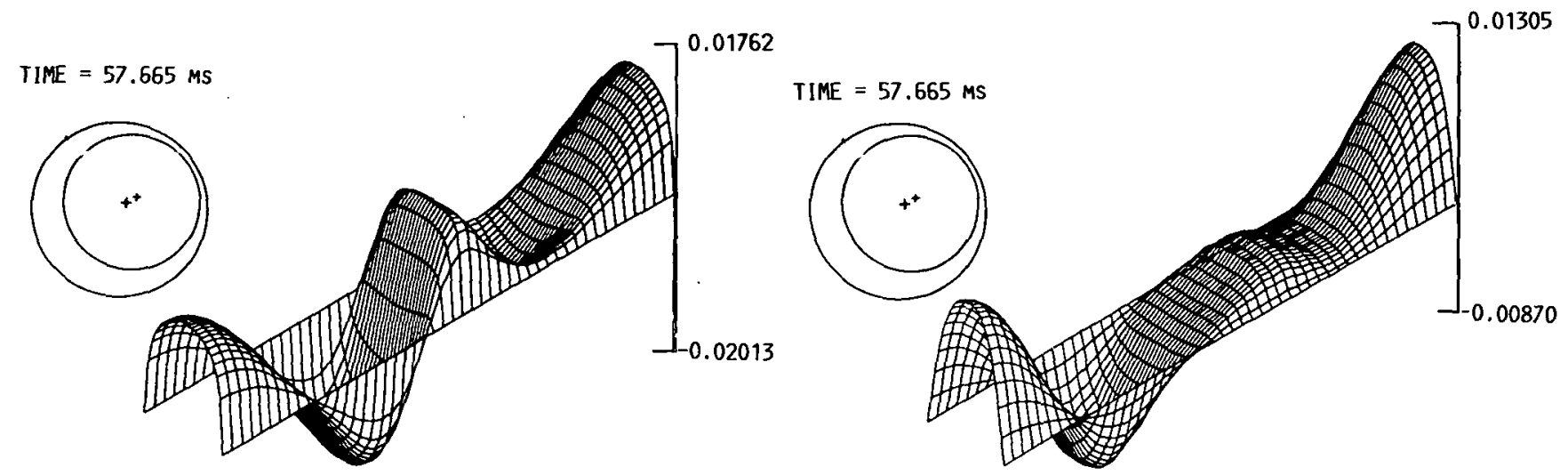

(A-1) INITIAL RELAXATION.

(A-2) AFTER 1 CYCLE.

(A) WITHOUT CAVITATION.

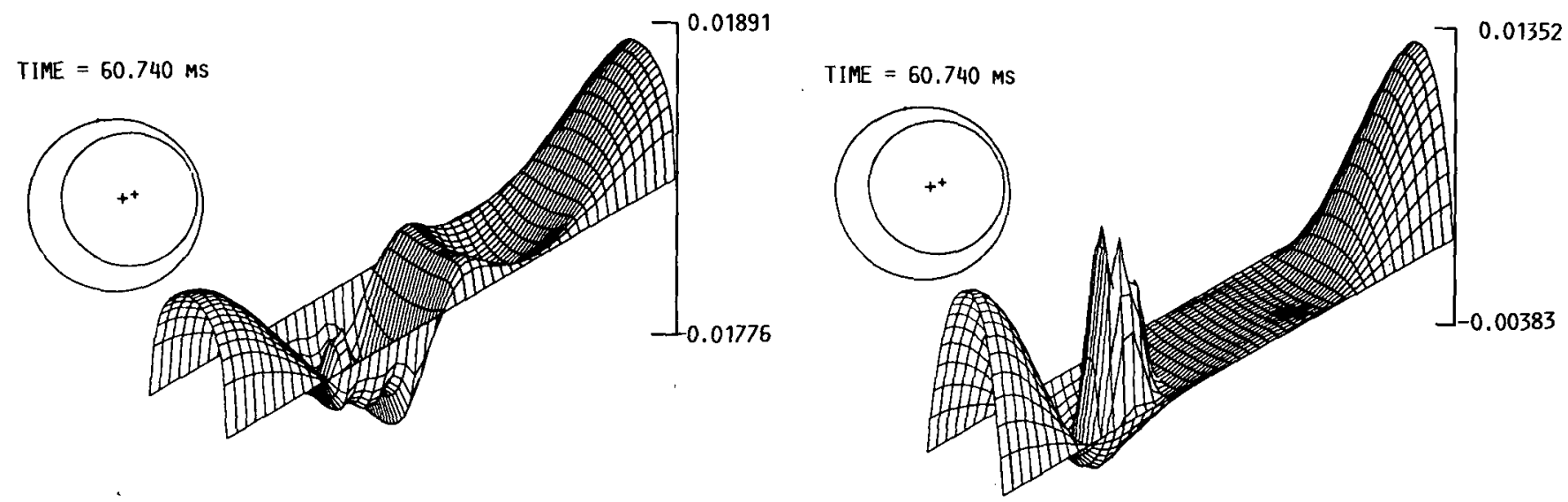

(B-1) INITIAL RELAXATION.

(B-2) AFTER 1 CYCLE.

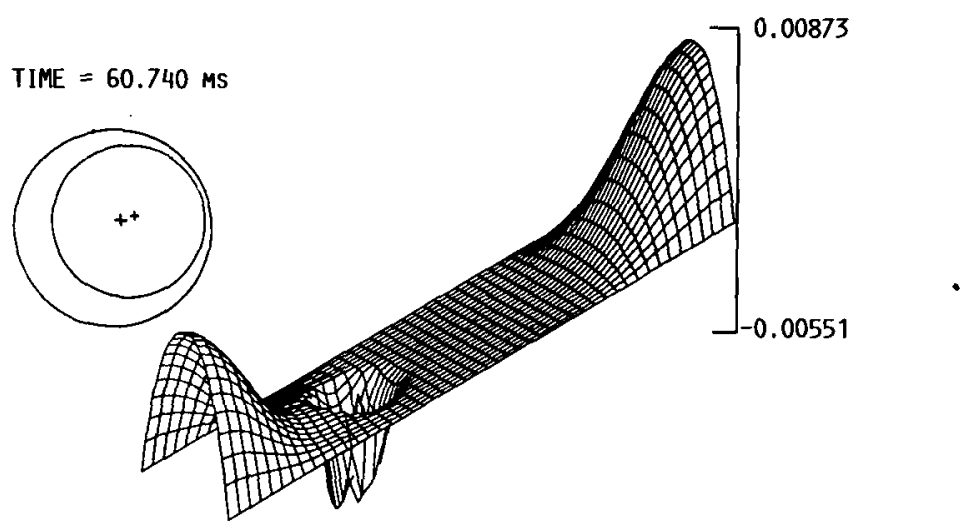

(B-3) AFTER 2 CYCLES.

(B) WITH CAVITATION.

FIGURE 4. - SMOOTH VERSUS UNSMOOTH RESIDUALS. 

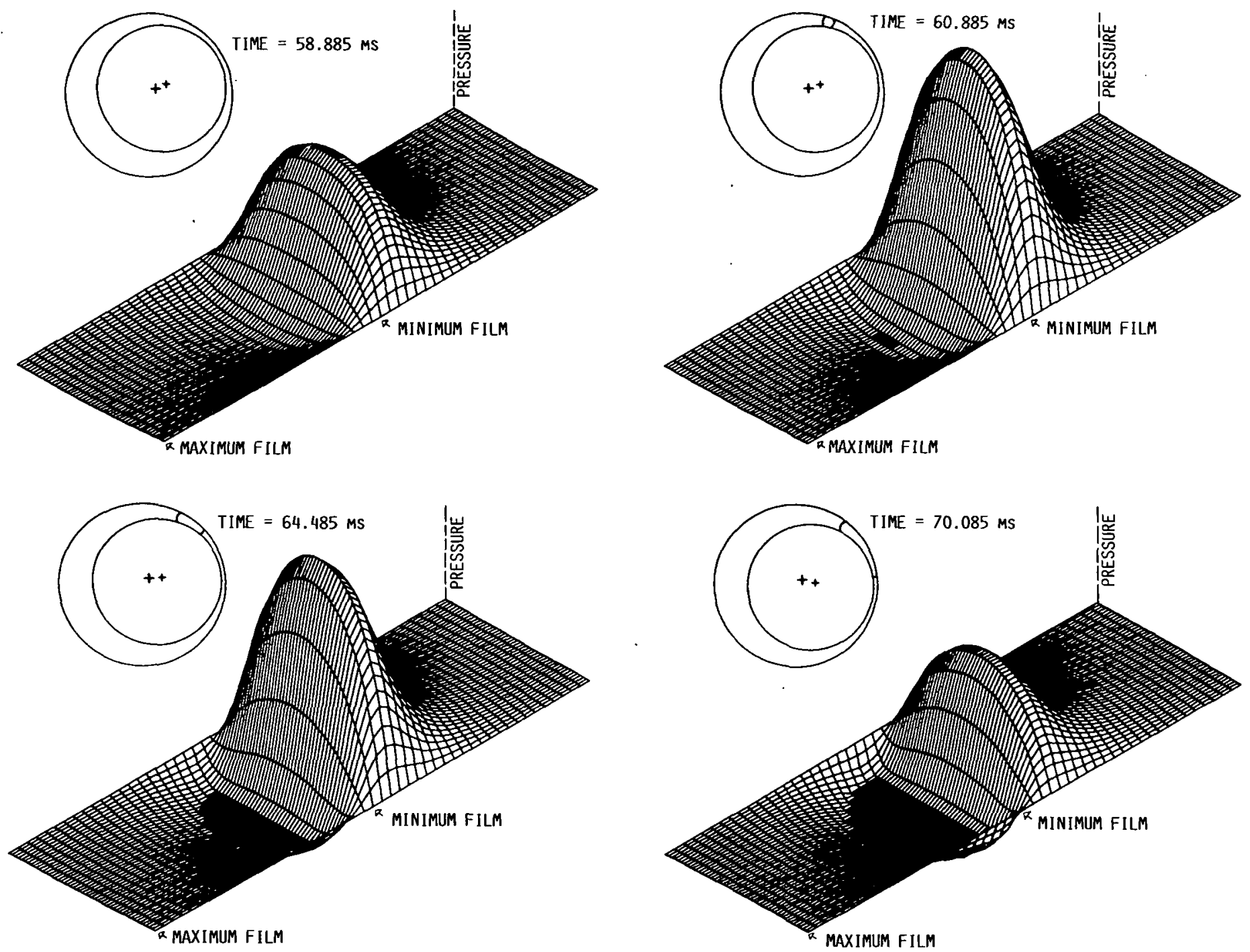

FIGURE 5. - PRESSURE DISTRIBUTION AT VARIOUS TIME STEPS. 


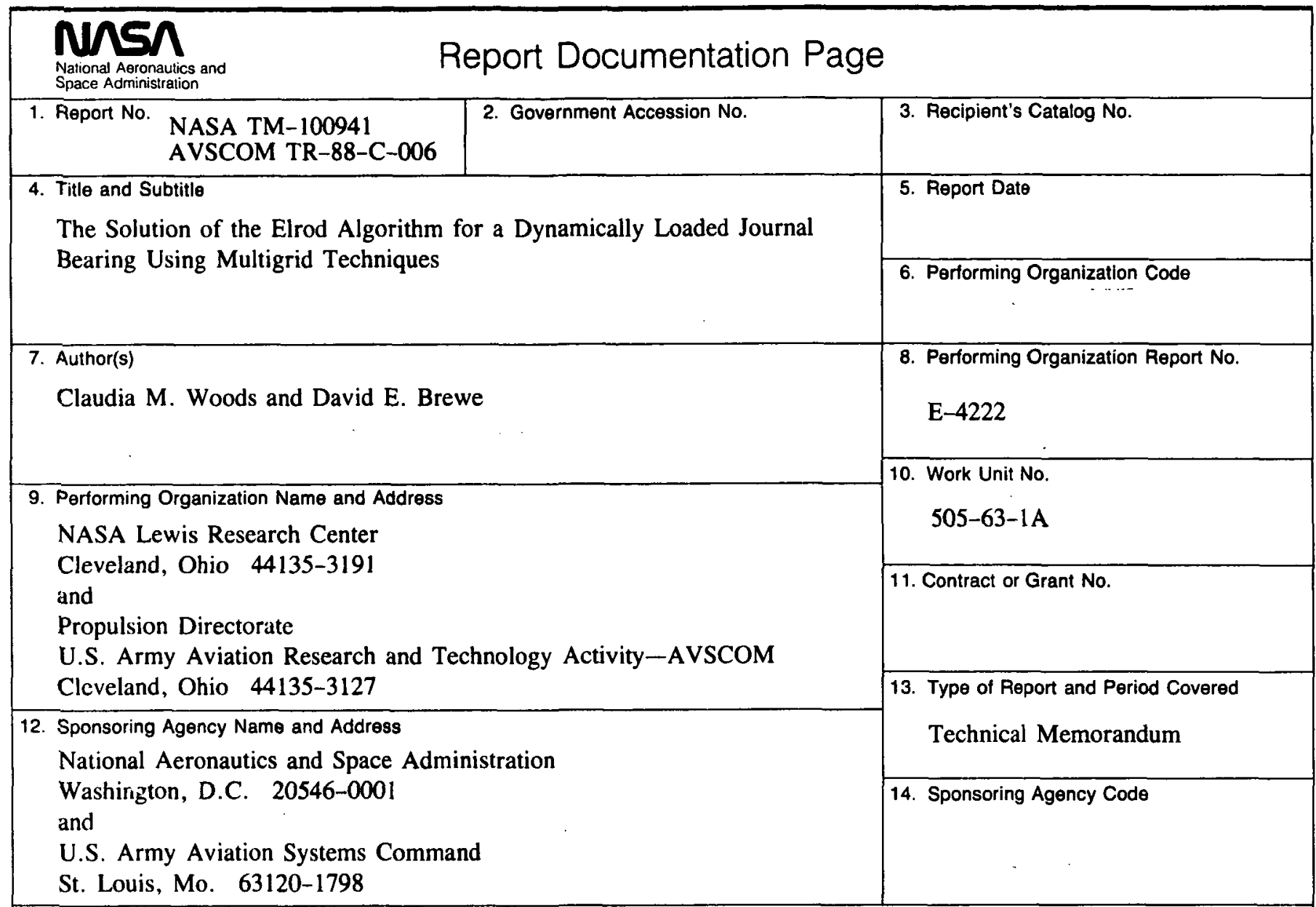

15. Supplementary Notes

Prepared for the 1988 Tribology Conference cosponsored by the ASME and STLE, Baltimore, Maryland, October 16-19, 1988. Claudia M. Woods, NASA Lewis Research Center, Cleveland, Ohio 44135; David E. Brewe, Propulsion Directorate, U.S. Army Aviation Research and Technology Activity-AVSCOM, Lewis Research Center, Cleveland, Ohio 44135.

16. Abstract

Numerical solution to a theoretical model of vapor cavitation in a dynamically loaded journal bearing is developed, utilizing a multigrid iterative technique. The method is compared with a noniterative approach in terms of computational time and accuracy. The computational model is based on the Elrod algorithm, a control volume approach to the Reynolds equation which mimics the Jakobsson-Floberg and Olsson cavitation theory. Besides accounting for a moving cavitation boundary and conservation of mass at the boundary, it also conserves mass within the cavitated region via a smeared mass or striated flow extending to both surfaces in the film gap. The mixed nature of the equations (parabolic in the full film zone and hyperbolic in the cavitated zone) coupled with the dynamic aspects of the problem create interesting difficulties for the present solution approach. Emphasis is placed on the methods found to eliminate solution instabilities. Excellent results are obtained for both accuracy and reduction of computational time.

17. Key Words (Suggested by Author(s))

Multigrid; Journal bearing; Cavitation; Dynamic loads; Bearing; Algorithm; Two-phase flow
18. Distribution Statement

Unclassified-Unlimited

Subject Category 34

\begin{tabular}{|c|c|c|c|}
\hline $\begin{array}{c}\text { 19. Security Classif. (of this report) } \\
\text { Unclassified }\end{array}$ & $\begin{array}{c}\text { 20. Security Classif. (of this page) } \\
\text { Unclassified }\end{array}$ & $\begin{array}{c}\text { 21. No of pages } \\
30\end{array}$ & $\begin{array}{c}\text { 22. Price* } \\
\mathbf{A 0 3}\end{array}$ \\
\hline
\end{tabular}


National Aeronautics and

Space Administration

Lewis Research Center

Cleveland. Ohio 44135

Orickal Businoss

Penaly for Pitrate Uso $\$ 000$
SECOND CLASS MAIL

ADDRESS CORRECTION REQUESTED

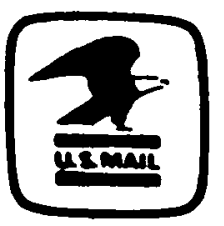

Postage and Fees Pald National Aeronautics and Spaco Administration NASA-451 